\title{
Shearing Nanometer-Thick Confined Hydrocarbon Films: Friction and Adhesion
}

\author{
Sivebæk, I. M.; Persson, B. N. J.
}

Published in:

Tribology Letters

Link to article, DOI:

10.1007/s11249-016-0656-0

Publication date:

2016

Document Version

Peer reviewed version

Link back to DTU Orbit

Citation (APA):

Sivebæk, I. M., \& Persson, B. N. J. (2016). Shearing Nanometer-Thick Confined Hydrocarbon Films: Friction and Adhesion. Tribology Letters, 62(5). https://doi.org/10.1007/s11249-016-0656-0

\section{General rights}

Copyright and moral rights for the publications made accessible in the public portal are retained by the authors and/or other copyright owners and it is a condition of accessing publications that users recognise and abide by the legal requirements associated with these rights.

- Users may download and print one copy of any publication from the public portal for the purpose of private study or research.

- You may not further distribute the material or use it for any profit-making activity or commercial gain

- You may freely distribute the URL identifying the publication in the public portal

If you believe that this document breaches copyright please contact us providing details, and we will remove access to the work immediately and investigate your claim. 


\title{
Shearing nanometer-thick confined hydrocarbon films: friction and adhesion
}

\author{
I.M. Sivebaek · B.N.J. Persson
}

the date of receipt and acceptance should be inserted later

\begin{abstract}
We present Molecular Dynamics (MD) friction and adhesion calculations for nanometer-thick confined hydrocarbon films with molecular lengths 20, 100 and 1400 carbon atoms. We study the dependency of the frictional shear stress on the confining pressure and sliding speed. We present results for the pull-off force as a function of the pull-off speed and the sliding speed. Some of the results are analyzed using the simple cobblestone model and good semi-quantitative agreement between the model predictions and the MD results are found.
\end{abstract}

Keywords Tribology $\cdot$ Adhesion $\cdot$ Molecular Dynamics $\cdot$ Nanometre hydrocarbon films

\section{Introduction}

The nature of fluids or soft solids confined between solids walls at nanometer separation is a central problem in tribology $[1,2]$. It has been shown that simple fluids such as linear alcanes, or fluids consisting of (nearly) spherical molecules such as squalane, when confined at short separation between flat solid walls (wetted by the fluid) form layers parallel to the solid walls. Usually, as the squeezing pressure increases, one layer after another of the confined fluid is squeezed out from the interface $[3,4]$, and the frictional shear properties of the interface changes in a discontinuous (quantized) manner as the number of confined monolayers changes.

I.M. Sivebaek

PGI, FZ-Jülich, 52425 Jülich, Germany

Novo Nordisk A/S, Device R\&D, DK-3400 Hillerod, Denmark

Mech. Eng. Dept., Technical University of Denmark, DK-2800 Lyngby, Denmark

Tel.: +4530751499

E-mail: ims@mek.dtu.dk

B.N.J. Persson

PGI, FZ-Jülich, 52425 Jülich, Germany 
In this paper we present new results for the properties of thin confined hydrocarbon films. Using Molecular Dynamics (MD) we have studied the friction and adhesion for nanometer-thick confined hydrocarbon films with molecular lengths of 20 , 100 and 1400 carbon atoms. In the bulk these hydrocarbons are all in the solid state at room temperature (the melting temperature for the $\mathrm{C}_{20} \mathrm{H}_{42}$ solid is $\approx 36^{\circ} \mathrm{C}$ and our MD simulations are for $T=300 \mathrm{~K}$ or $27^{\circ} \mathrm{C}$ ). We study the dependency of the frictional shear stress on the confining pressure and sliding speed. We present results for the pull-off force as a function of the pull-off speed and the sliding speed. Some of the results are analyzed using the simple cobblestone model and good semi-quantitative agreement between the model predictions and the MD results is found. For earlier related studies see [5-9].

\section{Molecular Dynamics model}

Linear alkanes $\mathrm{C}_{n} \mathrm{H}_{2 n+2}$ (with $n=20,100$ and 1400) were used as "lubricant" in the present calculations. The $\mathrm{CH}_{2} / \mathrm{CH}_{3}$ beads are treated in the united atom representation $[10,11]$. The Lennard-Jones potential was used to model the interaction between beads of different chains

$$
U(r)=4 \varepsilon_{0}\left[\left(\frac{r_{0}}{r}\right)^{12}-\left(\frac{r_{0}}{r}\right)^{6}\right]
$$

and the same poten-tial with modi-fied parameters $\left(\varepsilon_{1}, r_{1}\right)$ was used for the interaction of each bead with the substrate and block atoms.

The parameters were $\varepsilon_{0}=5.12 \mathrm{meV}$ for both the interior and the end beads, and $r_{0}=3.905 \AA$. For the interactions within the $\mathrm{C}_{n} \mathrm{H}_{2 n+2}$ molecules we used the standard OPLS model $[10,11]$, including flexible bonds, bond bending and torsion interaction, which results in bulk properties in good agreement with experimental data far below the boiling point [12]. Atomic mass 14 (for interior $\mathrm{CH}_{2}$ beads) and 15 (for the $\mathrm{CH}_{3}$ end groups) were used. Within a $\mathrm{C}_{n} \mathrm{H}_{2 n+2}$ chain we assume nearest neighbor $\mathrm{C}$ atoms are connected via springs with the spring constant $k$, which was chosen equal to $10 \mathrm{~N} / \mathrm{m}$. Note that this value is one order of magnitude smaller then the optimized $450 \mathrm{~N} / \mathrm{m}$ [10], and was chosen such to facilitate a reasonable time step of $1 \mathrm{fs}$. We used an angle bending interaction of the form $E(\cos \theta)=1 / 2 k_{\text {bend }}\left(\cos \theta-\cos \theta_{0}\right)$ with $k_{\text {bend }}=62543 \mathrm{~K}$ and $\theta_{0}=2.0001 \mathrm{rad}$. For the dihedral interaction we used the functional form in term of a cosine Fourier series $E(\phi) / k_{B}=\sum_{i=0}^{3} c_{i} \cos ^{i}(\phi)$ with parameters $c_{0}=1009.99 \mathrm{~K}, c_{1}=2018.95 \mathrm{~K}, c_{2}=136.37 \mathrm{~K}, c_{3}=-3165.30 \mathrm{~K}$. Internal beads of separation greater than 3 units are treated similarly as beads from different chains. The number of molecules was equal to 1000,200 and 14 for the $\mathrm{C}_{20} \mathrm{H}_{42}$, $\mathrm{C}_{100} \mathrm{H}_{202}$ and $\mathrm{C}_{1400} \mathrm{H}_{2802}$ systems respectively. For simplicity we denote these systems as $\mathrm{C} 20, \mathrm{C} 100$ and $\mathrm{C} 1400$ in what follows. The hydrocarbon films at room temperature consisted of 6 to 8 monolayers of molecules between the solid surfaces.

We have chosen the polymer-wall interaction potential to be so strong that no slip occurs at these interfaces. This is the case with $r_{1}=2.92 \AA, \varepsilon_{1}=160 \mathrm{meV}$. The lattice spacings of the block and of the substrate are $a=b=2.6 \AA$. 


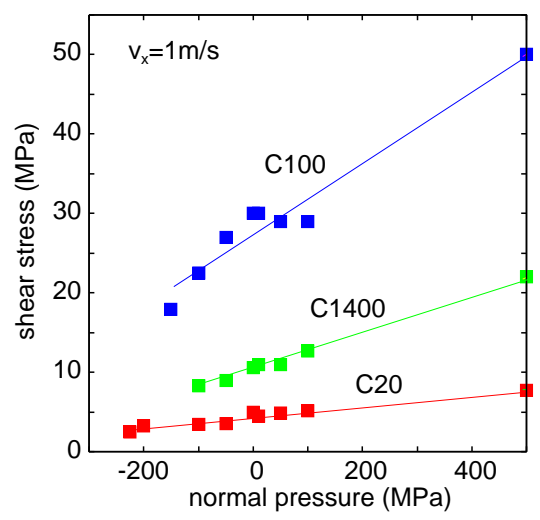

Fig. 1 The shear stress as a function of the normal pressure for $\mathrm{C}_{20} \mathrm{H}_{42}, \mathrm{C}_{100} \mathrm{H}_{202}$ and $\mathrm{C}_{1400} \mathrm{H}_{2802}$ systems at $\mathrm{vx}=1 \mathrm{~m} / \mathrm{s}$.

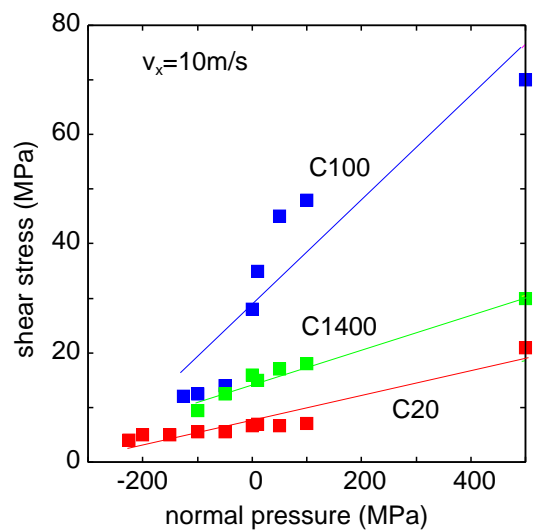

Fig. 2 The shear stress as a function of the normal pressure for $\mathrm{C}_{20} \mathrm{H}_{42}, \mathrm{C}_{100} \mathrm{H}_{202}$ and $\mathrm{C}_{1400} \mathrm{H}_{2802}$ systems at $\mathrm{vX}=10 \mathrm{~m} / \mathrm{s}$.

In our simulations heat is removed by the wall atoms which are kept at the imposed temperature by a thermostat of the Langevin type. That is, on the wall atoms act a fluctuating force and a damping choosen such as to give the imposed temperature. Because of the very short thermal diffusion distance from the center of the hydrocarbon slab to the walls, the temperature is very close to the thermostat temperature everywhere.

\section{Numerical results and analysis}

3.1 Frictional shear stress as a function of pressure and sliding speed

In this section we study how the frictional shear stress $\tau$ for confined hydrocarbon films depends on the nominal contact pressure $P$ and on the chain length C20, C100 


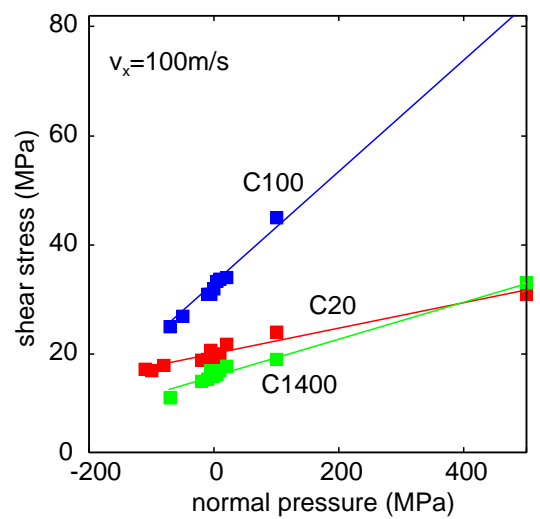

Fig. 3 The shear stress as a function of the normal pressure for $\mathrm{C}_{20} \mathrm{H}_{42}, \mathrm{C}_{100} \mathrm{H}_{202}$ and $\mathrm{C}_{1400} \mathrm{H}_{2802}$ systems at $\mathrm{vx}=100 \mathrm{~m} / \mathrm{s}$.

Table 1 The slope $k$ and adhesive pressure $P_{\mathrm{a}}$ defined by the equation $\tau=k\left(P-P_{\mathrm{ad}}\right)$ for the $\mathrm{C} 20, \mathrm{C} 100$ and $\mathrm{C} 1200$-systems. $P_{\text {detach }}$ is the force per unit surface area to separate the surfaces.

\begin{tabular}{l||c|c|c|c|}
\multicolumn{1}{|l||}{ system C20 } & \multicolumn{5}{l|}{} \\
\hline$v_{x}(\mathrm{~m} / \mathrm{s})$ & 0 & 1 & 10 & 100 \\
\hline \hline$k$ & & 0.007 & 0.022 & 0.023 \\
\hline$P_{\mathrm{a}}(\mathrm{MPa})$ & & -623 & -340 & -867 \\
\hline$P_{\text {detach }}(\mathrm{MPa})$ & -270 & -225 & -225 & -110 \\
\hline \hline \multicolumn{1}{|l||}{ system C100 } & \multicolumn{5}{l}{} \\
\hline$v_{x}(\mathrm{~m} / \mathrm{s})$ & 0 & 1 & 10 & 100 \\
\hline \hline$k$ & & 0.045 & 0.096 & 0.104 \\
\hline$P_{\mathrm{a}}(\mathrm{MPa})$ & & -608 & -296 & -312 \\
\hline$P_{\text {detach }}(\mathrm{MPa})$ & -180 & -150 & -125 & -70 \\
\hline \hline \hline system C1400 & \multicolumn{5}{|l}{} \\
\hline$v_{x}(\mathrm{~m} / \mathrm{s})$ & & 1 & 10 & 100 \\
\hline \hline$k$ & & 0.023 & 0.032 & 0.034 \\
\hline$P_{\mathrm{a}}(\mathrm{MPa})$ & & -453 & -453 & -472 \\
\hline$P_{\text {detach }}(\mathrm{MPa})$ & -180 & -100 & -100 & -70 \\
\hline
\end{tabular}

and C1400. In an earlier paper we presented this relation for one system, here we include all systems and include negative values for the normal pressure [13].

Fig. 1, 2 and 3 shows results for a $h \approx 3 \mathrm{~nm}$ thick confined films for the sliding speeds $v_{x}=1,10$ and $100 \mathrm{~m} / \mathrm{s}$, respectively. We note that to a good approximation the shear stress increases linearly with the contact pressure. The strait lines in the figures are given by

$$
\tau=k\left(P-P_{\mathrm{ad}}\right),
$$

where the slope parameter $k$ and the adhesive pressure $P_{\text {ad }}$ are given in Table 1 .

In figures 1 and 2 it can be observed that the linearity between the shear stress and the normal pressure is questionable for $\mathrm{C} 100$. This is due to an instability in the number of monolayers, which fluctuate between six and seven. Increasing the sliding 


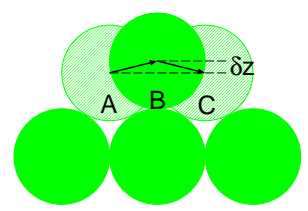

Fig. 4 The fluctuation $\delta z$ in the height as a spherical particle (molecular segment) moves from one bridge position A to another bridge position $\mathrm{C}$ via the top position $\mathrm{B}$. The work against the external pressure $P$ to bring the system to the top of the barrier equals $P \delta z$ (times the area occupied by the particle) and is assumed fully lost (probably due to emission of phonons) as the particle (rapidly) moves "downhill" from $\mathrm{B}$ to $\mathrm{C}$.

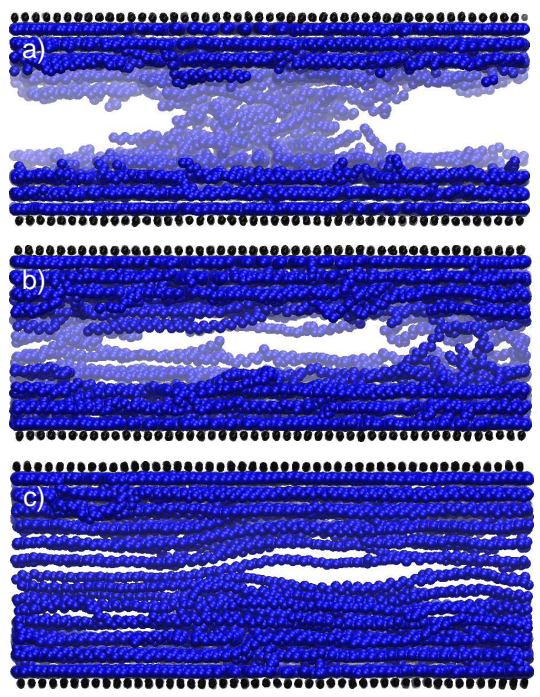

Fig. 5 Snapshots of the films of a) $\mathrm{C}_{20} \mathrm{H}_{42}$, b) $\mathrm{C}_{100} \mathrm{H}_{202}$ and c) $\mathrm{C}_{1400} \mathrm{H}_{2802}$. The sliding velocities are 100 $\mathrm{m} / \mathrm{s}$ and $1 \mathrm{~m} / \mathrm{s}$ in the $\mathrm{x}$ and $\mathrm{z}$ directions respectively. The surfaces have been separated by about $2 \mathrm{~nm}$.

velocity results in seven monolayers. Reducing the sliding velocity makes the film collaps to six monolayers. More details can be found in reference in [14].

In the Table we also give the detachment pressure or stress $P_{\text {detach }}$, which is the the pull-stress (i.e., most negative stress) for which the confined film is stable, i.e., the most negative pressure data points in Fig. 1, 2 and 3. In the Table we also give $P_{\text {detach }}$ for the case of no sliding $\left(v_{x}=0\right)$ as obtained during pull-off (at the speed $v_{z}=0.1 \mathrm{~m} / \mathrm{s}$ ), see Fig. 7 .

The adhesive pressure $P_{\text {ad }}$ can be estimated theoretically as follows [15,9]. Assume that the hydrocarbon chains form horizontal layers. Assume the layers interact only via the attractive van der Waals interaction plus a hard-wall repulsion when the layer separation is $d_{0} \approx 0.4 \mathrm{~nm}$. In this case the interaction potential between two hydrocarbon slabs with the separation $z$ is $V(z)=-w\left(d_{0} / z\right)^{2}$ for $z>d_{0}$, where $w$ is the work of adhesion. The adhesion pressure is the attractive force per unit surface area at the equilibrium separation $z=d_{0}$ so that $P_{\mathrm{ad}} \approx-V^{\prime}(z)=-2 w d_{0}^{2} / z^{3}$ for $z=d_{0}$ or $P_{\mathrm{ad}} \approx-2 w / d_{0}$. Using the work of adhesion $w \approx 0.1 \mathrm{~J} / \mathrm{m}^{2}$ and the equilibrium sepa- 


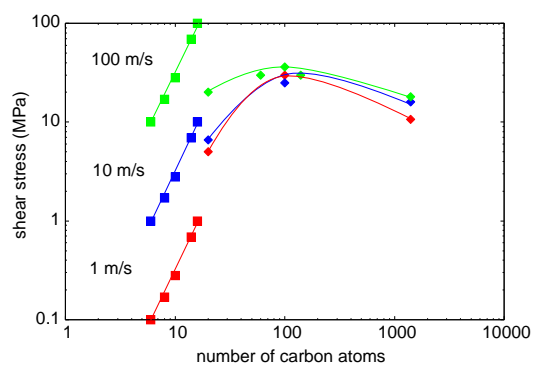

Fig. 6 The shear stress as a function of the length of the molecules expressed as the number of carbon atoms. The red, blue and green curves are for sliding velocities of $v_{x}=1,10$ and $100 \mathrm{~m} / \mathrm{s}$. The shear stresses up to $\mathrm{C} 16$ are calculated from measured bulk viscosity's. For C20 and up the points are from the MD-simulations at the confining pressure $P=10 \mathrm{MPa}$.

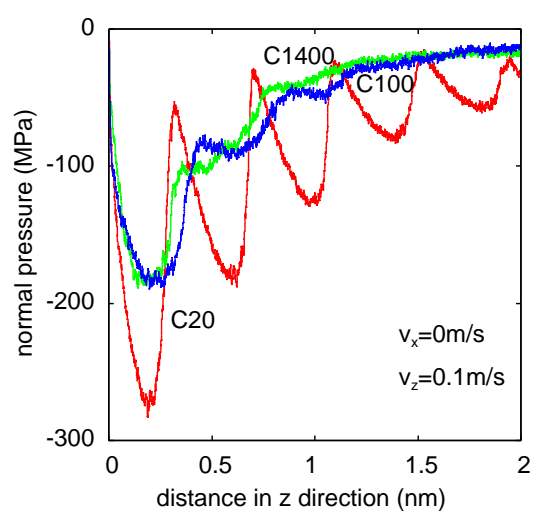

Fig. 7 The normal pressure as a function of the separation at $\mathrm{vz}=0.1 \mathrm{~m} / \mathrm{s}$ for the $\mathrm{C} 20, \mathrm{C} 100$ and $\mathrm{C} 1400$ systems. There is no sliding in the $x$-direction during separation.

ration $d_{0} \approx 0.4 \mathrm{~nm}$ gives $P_{\mathrm{ad}} \approx-500 \mathrm{MPa}$ which is very similar to what we obtained in the MD simulations (see Table 1).

Let us now briefly address the physical origin of the slope parameter $k$. Assume again that the hydrocarbons form well defined layers parallel to the solids walls. During sliding one layer slip relative to a nearby layer (see Fig. 4). The slip involves small fluctuations in the interlayer separation which is the origin of the dependency of the shear stress $\tau$ on the contact pressure $P$. Thus if the interlayer separation increases by $\delta z$ as the two layers displace with a distance $a$ of order half the intermolecular separation $a \approx d_{0} / 2$, and if we assume all the energy necessary to bring the system to the top of the barrier is lost as the layer slips downhill, we get $\tau d_{0} \approx P \delta z$ or $k=\delta z / d_{0}$. Assuming that the layers consist of a triangular (or hexagonal) lattice of spherical particles with radius $R=d_{0} / 2$, and that $\delta z$ is the height difference of a particle between a bridge and a hollow binding position, one gets $\delta z=\left[(4-1)^{1 / 2}\right.$ $\left.(4-4 / 3)^{1 / 2}\right] R \approx 0.099 R \approx 0.05 d_{0}$. Thus we predict $k \approx 0.05$ which is within the range of observed values for $k$ (see Table 1 ). 
As shown in Table 1 the magnitude (absolute value) of the adhesive pressure is larger than the force per unit area to break the contact (the detachment pressure $\left.P_{\text {detach }}\right)$. This is expected because $P_{\mathrm{a}}=P_{\text {detach }}$ only if all the bonds break simultaneously at the separating interface. For a macroscopic system this is never the case, where the bond-breaking usually occur via interfacial crack propagation. Because of stress concentration at the crack tip $P_{\mathrm{a}}>>P_{\text {detach }}$. For small systems the bond breaking process is more uniform [16] but the present simulation results show that even for systems with lateral dimensions of order $\sim 10 \mathrm{~nm}$ the bond breaking occur nonuniformly resulting in a detachment stress 4-8 times smaller than the stress expected if the interfacial bonds would break simultaneously at the separating interface.

Note that the detachment stress when no lateral sliding occur is larger than when lateral sliding occur (see Table 1). Thus we will show below that if the surfaces are separated by $v_{z}=0.1 \mathrm{~m} / \mathrm{s}$ but with $v_{x}=0$, the detachment stress $\approx-270 \mathrm{MPa}$ for C20 and about $\approx-180 \mathrm{MPa}$ for $\mathrm{C} 100$ and $\mathrm{C} 1400$. Thus also in these cases the magnitude (absolute value) of the adhesive pressure is larger than the force per unit area to break the contact (the detachment pressure $P_{\text {detach }}$ ).

It is interesting to compare the shear stress obtained for the confined hydrocarbon with the result expected from bulk viscosity data. The hydrocarbon systems we study are all in the solid state at room temperature. In Fig. 6 we therefore compare the MD results with the calculated shear stress $\tau=\eta \nu / h$ for C6-C16 which are all in the liquid state at room temperature. In all cases the hydrocarbon film thickness $h \approx 3 \mathrm{~nm}$.

Fig. 6 shows the shear stress as a function of the length of the molecules expressed as the number of carbon atoms. The red, blue and green curves are for sliding velocities of $v_{x}=1,10$ and $100 \mathrm{~m} / \mathrm{s}$. The shear stresses for C6-C16 are calculated from measured bulk viscosities. For $\mathrm{C} 20-\mathrm{C} 1400$ the points are from the MD-simulations (from Fig. 1, 2 and 3 ) at the confining pressure $P=10 \mathrm{MPa}$. Note that the variation in the frictional shear stress with the sliding speed for the confined films (MD-results) is much smaller than expected from extrapolating the bulk viscosity data for the C6C16 systems. This is in accordance with experimental observations [17] and earlier MD simulations [18] for confined systems where it was found $\tau \sim \dot{\gamma}^{1-n}$ (where the shear rate $\dot{\gamma}=v / h$ ) with $n \approx 0.9$ at room temperature (corresponding to an effective viscosity $\eta_{\text {eff }} \sim \dot{\gamma}^{-n}$, e.g. a "fluid" undergoing strong shear thinning) .

For elastic solids with randomly rough surfaces, and assuming non-adhesive contact, the area of real contact $A$ is given by [19-22]

$$
P_{0}=\frac{\xi}{\kappa} E^{*} \frac{A}{A_{0}}
$$

where $\kappa \approx 2$ and where $\xi^{2}=\left\langle|\nabla h|^{2}\right\rangle$ is the surface mean-square-slope and $P_{0}$ the nominal contact pressure. Using that $P_{0} A_{0}=F_{\mathrm{N}}$, where $F_{\mathrm{N}}$ is the (normal) nominal squeezing pressure, we get

$$
F_{\mathrm{N}}=\frac{\xi}{\kappa} E^{*} A
$$

where $E^{*}$ is the effective Young's modulus. Using (2) the friction force

$$
F_{\mathrm{f}}=A \tau=A k\left(P-P_{\mathrm{ad}}\right)=k F_{\mathrm{N}}-k A P_{\mathrm{ad}},
$$


where we have used that the normal (squeezing) force $F_{\mathrm{N}}=A P$. Next using (4) gives

$$
F_{\mathrm{f}}=k F_{\mathrm{N}}-\frac{k \kappa}{\xi E^{*}} P_{\mathrm{ad}} F_{\mathrm{N}}=k\left(1-\frac{\kappa P_{\mathrm{ad}}}{\xi E^{*}}\right) F_{\mathrm{N}}
$$

and the friction coefficient

$$
\mu=k\left(1+\frac{\kappa\left|P_{\mathrm{ad}}\right|}{\xi E^{*}}\right)
$$

For hydrocarbones (see above) $\kappa\left|P_{\mathrm{ad}}\right| \approx 1 \mathrm{GPa}$, which should also hold for other soft materials where the molecules interact with similar weak interactions as for the hydrocarbons. If we assume $\xi \approx 1$, as it is typical for many natural and engineering surfaces[23], we conclude that the adhesive contribution will dominate for soft materials like rubber (with $E^{*} \approx 10$ or less, when perturbed at low frequencies). For stiffer materials like polymers in the glassy state, where $E^{*} \approx 1 \mathrm{GPa}$, the two contributions in (5) will typically be of similar importance. However, for most non-rubber materials, if $\xi \approx 1$ plastic deformations will occur in the contact regions. In these case the local pressure will be of order the penetration hardness, which is typically $0.1 \mathrm{GPa}$ for polymers in the glassy state. Thus in this cases too, the adhesive pressure term will be very important.

For soft elastic solids when an adhesive interaction occur between the solids as in the case studied in this paper, the area of real contact will also increase in which case (3) with $\kappa \approx 2$ is no longer accurate. In this case one must distinguish between two cases[24-26]: (a) If the adhesive interaction is not too strong, (3) is still approximately valid, but $\kappa$ is larger than 2 . In this case, if adhesion hysteresis is negligible, no adhesion will be observed in a pull-off experiment. The physical origin of this is the elastic energy stored up in the highest asperities, which act as compressed springs and break the adhesive atomic bonds as the external load is reduced to zero. (b) If the adhesive force is strong enough, the contact area will be non-vanishing also when the external load vanish. In this case a finite force is necessary to remove the contact between the solids.

\subsection{Dependency of the pull-off force on the pull-off speed}

Fig. 7 and 8 show the variation in the normal pressure as a function of the pull-off distance for the pull-off speed $v_{z}=0.1$ and $10 \mathrm{~m} / \mathrm{s}$, respectively. For the shortest chain C20 strong oscillations occur in the pull-off force for $v_{z}=0.1 \mathrm{~m} / \mathrm{s}$, and the wall-wall interaction prevail to larger surface separations than for $v_{z}=10 \mathrm{~m} / \mathrm{s}$. This is due to the formation of a capillary bridge at the lower pull-off speed, and to (approximate) layering of the molecules in the capillary bridge [27]. Thus during pull-off the capillary bridge extends vertically by shrinking in width and adding more layers in the vertical direction [28]. At the separation velocity $v_{z}=10 \mathrm{~m} / \mathrm{s}$ almost no oscillations remains in the force-displacement curve for $\mathrm{C} 20$, and the pressure decay faster with the separation distance than for $v_{z}=0.1 \mathrm{~m} / \mathrm{s}$, indicating a different arrangement of the molecules in the junction. Clearly there exist some relaxation time $\tau^{*}$ such that if the separation time scale $t^{*} \approx h / v_{z}>>\tau^{*}$ the molecules in the junction can rearrange in an adiabatic way forming the free-energy minimum configuration, while in 


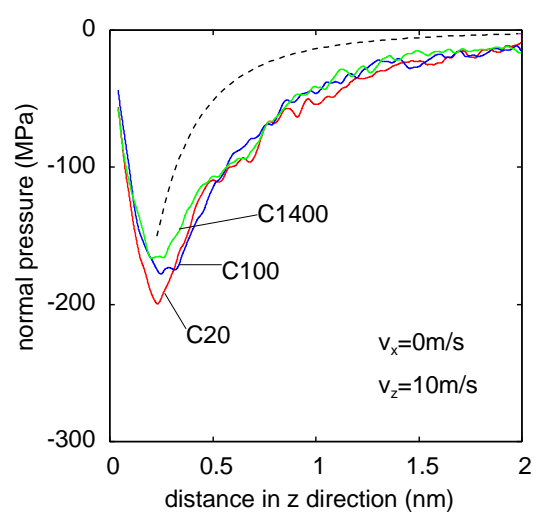

Fig. 8 The normal pressure as a function of the separation at $\mathrm{vz}=10 \mathrm{~m} / \mathrm{s}$ for the $\mathrm{C} 20, \mathrm{C} 100$ and $\mathrm{C} 1400$ systems. The dashed line indicate the van der Waals pressure dependency $\sim-(z+d)^{-3}$ (where $d=0.4$ $\mathrm{nm}$ is the equilibrium separation between two hydrocarbon layers) assuming the separation occur at a sharp flat interface. There is no sliding in the $x$-direction during separation.

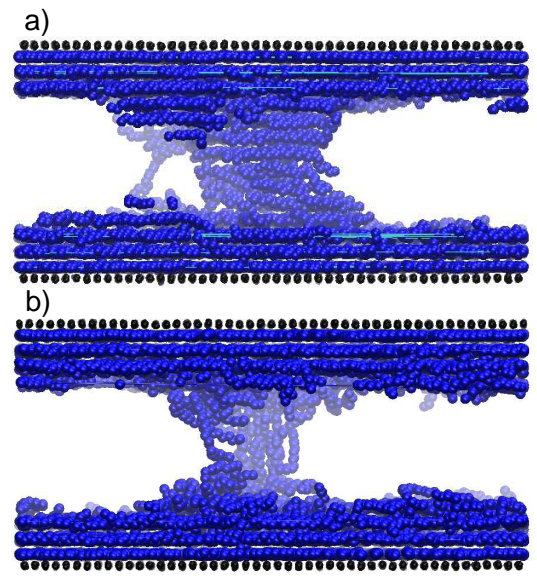

Fig. 9 Snapshot pictures of the C20-system during pull-off with the speed (a) $v_{z}=1 \mathrm{~m} / \mathrm{s}$ and (b) $10 \mathrm{~m} / \mathrm{s}$. The separation distance $\Delta h \approx 3 \mathrm{~nm}$. Note that at the lower separation speed a capillary bridge forms with the axis of the C20 molecules parallel to the solid walls, while at the higher separation speed the axis of the molecules are on the mainly orthogonal to the solid surfaces, bridging the gap between the surfaces as it is typical for crazing.

the limit $t^{*}<<\tau^{*}$ this is not the case. From the results above we estimate $\tau^{*} \approx 1 \mathrm{~ns}$ for C20.

Fig. 9 shows snapshot pictures of the C20-system during pull-off with the speed (a) $v_{z}=1 \mathrm{~m} / \mathrm{s}$ and (b) $10 \mathrm{~m} / \mathrm{s}$. The separation distance $\Delta h \approx 3 \mathrm{~nm}$. Note that at the lower separation speed a capillary bridge forms with the $\mathrm{C} 20$ molecules axis parallel to the solid walls, while at the higher separation speed the molecule axis is on the average orthogonal to the solid surfaces, bridging the gap between the surfaces as it is typical for crazing [29]. 


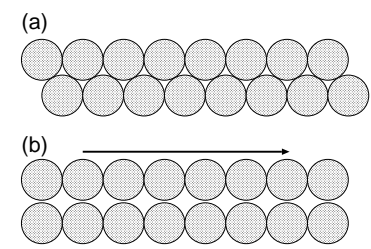

Fig. 10 Molecular arrangement during (a) stationary contact $\left(v_{x}=0\right)$ and (b) during sliding $\left(v_{x}>0\right)$. During sliding the (time-averaged) separation between the molecular layers increases. (Schematic.)

At the lowest pull-off speed the C100 and C1400-systems exhibit step-like changes (with a step width of order the interlayer separation) in the force-distance curves, while the force-distance curves are relative smooth at the higher pull-off speed. It is interesting to note that at the separation speed $10 \mathrm{~m} / \mathrm{s}$ all the three hydrocarbon films result in nearly the same pressure-distance $P(z)$-relation, but the decay of $P(z)$ for large $z$ is much slower than the $\sim-\left(z+d_{0}\right)^{-3}\left(d_{0} \approx 0.4 \mathrm{~nm}\right)$, expected if the separation would occur at a sharp, flat interface (see dashed line in Fig. 8) without connecting bridges.

\subsection{Dependency of the pull-off force on the sliding speed}

Compared to stationary contact, sliding result in the formation of more well defined layering, e.g., it reduces the entanglement between different layers. Thus, molecular links formed by molecules having part of the chain is in one layer and the other part in another layer, will tend to be broken by the sliding motion, at least at the interface where most of the slip occur (which for long hydrocarbon chains usually is a very well defined interface). At the same time the average separation between the molecular layers increases (see Fig. 10) resulting in a reduction in the (normal) force necessary to separate the surfaces. This effect is illustrated in Fig. 11 and 12, for the $\mathrm{C} 20$ and $\mathrm{C} 1400$ systems. The figures show the pull-off stress as a function of time during pull-off at the speed $v_{z}=1 \mathrm{~m} / \mathrm{s}$, and for the sliding speeds $v_{x}=1, v_{x}=10$ and $100 \mathrm{~m} / \mathrm{s}$.

\section{Summary and conclusion}

We have presented Molecular Dynamics (MD) friction and adhesion calculations for nanometer-thick confined hydrocarbon films with molecular lengths 20, 100 and 1400 carbon atoms, and studied the dependency of the frictional shear stress on the confining pressure and sliding speed. We have presented results for the pull-off force as a function of the pull-off speed and the sliding speed. Some of the results where analyzed using the simple cobblestone model and we found good semi-quantitative agreement between the model predictions and the MD results.

We have shown that the pull-off force force is lower in sliding systems, which is due to the formation of more well defined layers with larger average separation then in the absence of sliding. For C20 we observed that an increase in the pull-off 


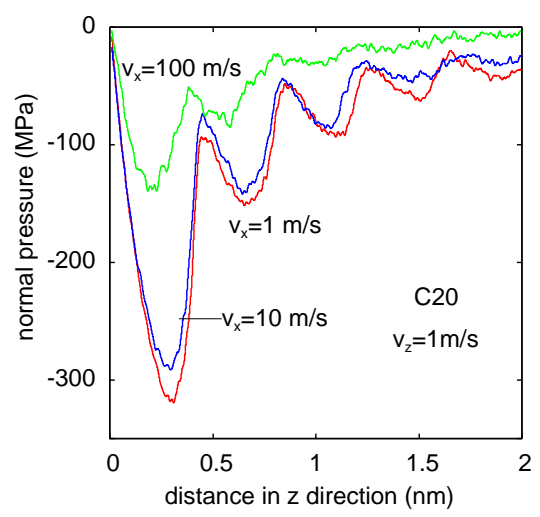

Fig. 11 The normal pressure as a function of the separation at $v z=1 \mathrm{~m} / \mathrm{s}$ for $\mathrm{C}_{20} \mathrm{H}_{42}$. Curves for sliding velocities of 1,10 and $100 \mathrm{~m} / \mathrm{s}$ in the $\mathrm{x}$ direction are shown.

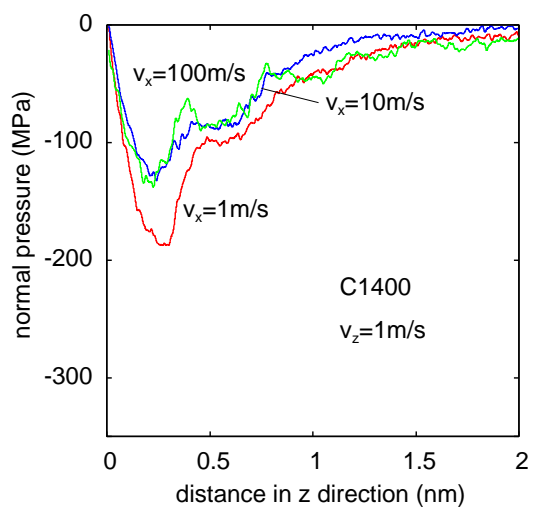

Fig. 12 (The normal pressure as a function of the separation at $\mathrm{vZ}=1 \mathrm{~m} / \mathrm{s}$ for $\mathrm{C}_{1400} \mathrm{H}_{2802}$. Curves for sliding velocities of 1,10 and $100 \mathrm{~m} / \mathrm{s}$ in the $\mathrm{x}$ direction are shown.

velocity resulted in a decrease in the pull-off force. We attribute this to the absence of well-defined capillary bridges at high pull-off speed.

Acknowledgements I.M.S. acknowledge support from IFF, FZ-Jülich, hospitality and help of the staff during their research visits.

The research work was performed within a Reinhart-Koselleck project funded by the Deutsche Forschungsgemeinschaft (DFG). We would like to thank DFG for the project support under the reference German Research Foundation DFG-Grant: MU 1225/36-1.

The research work was also supported by the DFG-grant: PE 807/10-1. This work is supported in part by COST Action MP1303.

\section{References}

1. B.N.J. Persson: Sliding Friction: Physical Principles and Applications, 2nd ed. (Springer, Heidelberg, 2000). 
2. E. Gnecco and E. Meyer, Elements of Friction Theory and Nanotribology, Cambridge University Press (2015).

3. B N J Persson and F Mugele: Squeeze-out and wear: fundamental principles and applications Journal of Physics: Condensed Matter 16, R295 (2004)

4. I M Sivebaek, V N Samoilov and B N J Persson: Squeezing molecular thin alkane lubrication films between curved solid surfaces with long-range elasticity: Layering transitions and wear, Journal of Chemical Physics 119, 3314 (2003)

5. Simulations of the kinetic friction due to adsorbed surface layers By: He, G; Robbins, MO TRIBOLOGY LETTERS Volume: 10 Issue: 1-2 Pages: 7-14 Published: JAN-MAR 2001

6. Adsorbed layers and the origin of static friction By: He, G; Muser, MH; Robbins, MO SCIENCE Volume: 284 Issue: 5420 Pages: 1650-1652 Published: JUN 41999

7. Solid friction from stick-slip down to pinning and aging By: Baumberger, Tristan; Caroli, Christiane ADVANCES IN PHYSICS Volume: 55 Issue: 3-4 Pages: 279-348 Published: MAY-JUN 2006

8. Frictional dissipation and interfacial glass transition of polymeric solids By: Bureau, Lionel; Caroli, Christiane; Baumberger, Tristan PHYSICAL REVIEW LETTERS Volume: 97 Issue: 22 Article Number: 225501 Published: DEC 12006

9. Simple microscopic theory of Amontons's laws for static friction By: Muser, MH; Wenning, L; Robbins, MO PHYSICAL REVIEW LETTERS Volume: 86 Issue: 7 Pages: 1295-1298 Published: FEB 12 2001

10. W.L. Jorgensen, J.D. Madura and C.J. Swenson: Optimized Intermolecular Potential Functions for Liquid Hydrocarbons, J. Am. Chem. Soc. 106, 6638 (1984)

11. D.K. Dysthe, A.H. Fuchs and B. Rousseau: Fluid transport properties by equilibrium molecular dynamics. III. Evaluation of united atom interaction potential models for pure alkanes, J. Chem. Phys. 112, 7581 (2000).

12. M.G. Martin and J.I. Siepmann: Transferable potentials for phase equilibria. 1. United-atom description of n-alkanes, J. Phys. Chem. B 102, 2569 (1998).

13. I M Sivebaek, V N Samoilov and B N J Persson: Frictional properties of confined polymers, European Physical Journal E 27, 37 (2008).

14. I M Sivebaek, V N Samoilov and B N J Persson: Velocity dependence of friction of confined hydrocarbons, Langmuir 26, 8721 (2010).

15. A. Berman, C. Drummond and J. Israelachvili: Amonton's law at the molecular level, Tribology Letters 4, 95 (1998).

16. B.N.J Persson: Nanoadhesion, Wear 254, 832 (2003).

17. S. Yamada: General shear-thinning dynamics of confined fluids Tribology Letters 13, 167 (2002).

18. I M Sivebaek, V N Samoilov and B N J Persson: Effective viscosity of confined hydrocarbons, Physical Review Letters 108(1), 1 (2012).

19. B.N.J. Persson: Contact mechanics for randomly rough surfaces, Surface Science Reports 61, 201 (2006).

20. S. Hyun, L. Pei, J.F. Molinari, M.O. Robbins: Finite-element analysis of contact between elastic selfaffine surfaces, Phys. Rev. E 70 (2004) 026117

21. C. Campana, M.H. Müser: Contact mechanics of real vs. randomly rough surfaces: A Green's function molecular dynamics study Europhys Lett 77, 38005 (2007)

22. V.A. Yastrebova, G. Anciaux, J.-F Molinari: From infinitesimal to full contact between rough surfaces: Evolution of the contact area 52, 83 (2015)

23. B.N.J. Persson, On the fractal dimension of rough surfaces Tribology Letters 54, 99 (2014).

24. B.N.J. Persson, I.M. Sivebaek, V.N. Samoilov, K. Zhao, A.I. Volokitin and Z. Zhang: On the origin of Amonton's friction law, J Phys Condens Matter 20395006 (2008).

25. L. Pastewka and M.O. Robbins: Contact between rough surfaces and a criterion for macroscopic adhesion PNAS 3298 (2014).

26. B.N.J. Persson and M. Scaraggi: Theory of adhesion: Role of surface roughness J. Chem. Phys. 141, 124701 (2014).

27. S. de Beer, E. Kutnyanszky, P.M. Schn, G.J. Vancso and M.H. Müser: Solvent-induced immiscibility of polymer brushes eliminates dissipation channels Nature Communications 5(May), 3 (2014).

28. V.A. Froltsov, M. Klppel and G. Raos: Molecular dynamics simulation of rupture in glassy polymer bridges within filler aggre-gates Physical Review E 86(4), 041801 (2012).

29. T. Ge, G.S. Grest and M.O. Robbins: Tensile Fracture of Welded Polymer Interfaces: Miscibility, Entanglements, and Crazing Macromolecules 47(19), 6982 (2014). 